\title{
THE ROLE OF NEWLY BORN MAGNETARS IN GAMMA-RAY BURST X-RAY AFTERGLOW EMISSION: ENERGY INJECTION AND INTERNAL EMISSION
}

\author{
Yun-Wei Yu ${ }^{1,2}$, K. S. Cheng ${ }^{1}$, AND Xiao-Feng CAO ${ }^{2}$ \\ ${ }^{1}$ Department of Physics, The University of Hong Kong, Pokfulam Road, Hong Kong, China; yuyw@hku.hk, hrspksc@hkucc.hku.hk \\ 2 Institute of Astrophysics, Huazhong Normal University, Wuhan 430079, China \\ Received 2010 January 31; accepted 2010 April 2; published 2010 April 29
}

\begin{abstract}
Swift observations suggest that the central compact objects of some gamma-ray bursts (GRBs) could be newly born millisecond magnetars. Therefore, considering the spin evolution of the magnetars against $r$-mode instability, we investigate the role of magnetars in GRB X-ray afterglow emission. Besides modifying the conventional energy injection model, we pay particular attention to the internal X-ray afterglow emission, whose luminosity is assumed to track the magnetic dipole luminosity of the magnetars with a certain fraction. Following a comparison between the model and some selected observational samples, we suggest that some so-called canonical X-ray afterglows including the shallow decay, normal decay, and steeper-than-normal decay phases could be internally produced by the magnetars (possibly through some internal dissipations of the magnetar winds), while the (energized) external shocks are associated with another type of X-ray afterglows. If this is true, then from those internal X-ray afterglows we can further determine the magnetic field strengths and the initial spin periods of the corresponding magnetars.
\end{abstract}

Key words: gamma-ray burst: general - stars: neutron

Online-only material: color figures

\section{INTRODUCTION}

Gamma-ray bursts (GRBs) are short, intense flashes of soft gamma rays $(\sim 0.01-1 \mathrm{MeV})$, which are always followed by long-lasting low-frequency afterglow emission. Usually, the afterglow emission is attributed to an external forward shock arising from the interaction of the GRB outflow with the circumburst medium, whereas the mechanisms responsible for the bursts are still under debate. Since the launch of the Swift spacecraft (Gehrels et al. 2004), many detailed features of the GRB X-ray afterglows have been revealed by the X-Ray Telescope (XRT) aboard the spacecraft. Then Nousek et al. (2006) and Zhang et al. (2006) phenomenologically summarized a "canonical" X-ray light curve (LC) with four smooth segments (sometimes superposed by some sharp flares), although the LCs owning all components are actually uncommon. Specifically, the four different emission phases they defined include: (1) Initial steep decay phase that is widely accepted to be the tail of the prompt emission (i.e., curvature effect; Fenimore et al. 1996; Kumar \& Panaitescu 2000); (2) Shallow decay (even a plateau) phase that is usually ascribed to a continuous energy injection into the external shock (Rees \& Mészáros 1998; Dai \& Lu 1998a, 1998b; Zhang \& Mészáros 2001); (3) Normal decay phase that does not contradict with the standard external shock model; and (4) Steeper-than-normal decay phase that is often connected to the jet break (Rhoads 1997). To summarize, in the conventional picture as described above, the emission during the shallow decay, normal decay, and steeper-than-normal decay phases (of interest in this paper) is usually considered to be associated with the external shock, while the initial steep decay emission as well as the flares is probably of internal origin.

The X-ray shallow-decay and flare emission strongly suggest that GRB central objects should have long activities after the bursts. Therefore, highly magnetized, rapidly spinning pulsars (i.e., millisecond magnetars) gradually become a popular candidate of the central compact objects (e.g., De Pasquale et al. 2007; Metzger et al. 2007; Troja et al. 2007; Zhang \& Dai 2008,
2009; Bucciantini et al. 2009; Corsi \& Mészáros 2009; Lyons et al. 2009), although the black hole model is still an attractive choice. It is a difficult task to observationally distinguish between the magnetar and black hole models. However, some magnetohydrodynamic simulations showed that the magnetar model could be more mature in the sense that it provides quantitative explanations for the durations, energies, Lorentz factors, and collimation of long GRB outflows (Metzger 2010). Moreover, with a spinning-down magnetar, some Swift-XRT features can be explained well (e.g., Dai et al. 2006; Fan \& Xu 2006; Yu \& Dai 2007). Specifically, the unusual X-ray afterglow LC of GRB 070110, where a nearly constant X-ray emission is followed by a very steep decline of $\alpha \sim 9$ (where $\alpha$ is the decline index of $t^{-\alpha}$ ), can be understood by ascribing the plateau emission to magnetar-driven internal emission (Troja et al. 2007).

The internal plateau emission of GRB 070110 also tells us that, in addition to the initial steep decay and flare phases, the $\mathrm{X}$-ray emission during any other afterglow phases could also arise from some internal dissipation mechanisms. Therefore, it is fair to consider that the long active central objects of some other GRBs could also play an essential, relatively more direct role in their afterglow emission, at least in the X-ray band. In some extreme situations, we suspect that such an internalorigin emission component could even dominate the total $\mathrm{X}$-ray afterglow emission of a GRB, whereas the external shock emission is outshined in the X-ray band. In other words, the conventional external shock model is only one choice among various afterglow origin models, as also proposed by some authors before (Ghisellini et al. 2007; Kumar et al. 2008; Cannizzo \& Gehrels 2009; Lindner et al. 2010; Lyutikov 2009). A wide investigation into the GRB X-ray afterglows given by Willingale el al. (2007) indeed showed that more than $100 \mathrm{X}$-ray afterglows can be divided into two emission components, one of which is probably of internal origin.

Following the observational results and the two theoretical considerations above, in this paper, we investigate in more 
detail the role of millisecond magnetars in the X-ray afterglow emission of some GRBs, based on a careful analysis on the spin evolution of the magnetars. Besides modifying the conventional energy injection model (Dai \& Lu 1998a, 1998b; Zhang \& Mészáros 2001), we pay particular attention to the internal X-ray afterglow emission that is produced by the magnetars, possibly through some internal dissipations in the magnetar winds. The luminosity of this internal emission is assumed to simply track the magnetic dipole luminosity of the magnetars with a certain fraction. To summarize, the observed X-ray afterglows could be emitted from two different regions (i.e., an internally dissipated magnetar wind and an energized external shock) at very different radii. The competition between these two emission components leads to a diversity of the X-ray afterglow LCs.

In Section 2, we briefly review the spin-down of magnetars against $r$-mode instability. We analyze the temporal behaviors of X-ray afterglows by combining the contributions from a magnetar wind and an external shock in Section 3, where the energy injection from the wind to the shock is also taken into account. In Section 4, some observational samples are selected and fitted in order to confront the model with observations. Meanwhile, some implications for the magnetars from the afterglow data are discussed. Finally, a summary and a discussion are given in Section 5.

\section{SPIN-DOWN OF MAGNETARS}

For long GRBs associated with Type Ibc supernovae, the central magnetars could be formed during the core collapse of massive, rotating stars, since powerful magnetocentrifugal outflows from the nascent magnetars may stave off black hole formation entirely (Metzger 2010). For short GRBs, the merger of compact binaries may also give rise to a massive neutron star if the equation of state of stellar matter is stiff enough, as implied by the observations of kilohertz quasi-periodic oscillations in accreting neutron stars (Kluźniak 1998; Kluźniak \& Ruderman 1998). Therefore, it seems somewhat acceptable that the central compact objects of some GRBs are millisecond magnetars. The idea that GRBs could originate from magnetars was actually proposed almost two decades ago (Usov 1992, 1994; Paczynski 1992; Duncan \& Thompson 1992), far before the Swift era. Specifically, several central engine mechanisms have been proposed, e.g., a neutrino-driven wind (Thompson 1994; Metzger et al. 2007; Bucciantini et al. 2009), a magnetic reconnection-accelerated wind (Drenkhahn 2002; Drenkhahn \& Spruit 2002), or a hyperaccretion onto the magnetars (Zhang \& Dai 2008, 2009). These violent processes lead the initial spin evolution of the magnetars during the first tens of seconds to be very complicated.

However, on the relatively longer (afterglow) timescales concerned here, the short-term processes mentioned above could be no longer important. Then the spin-down of the magnetars would be mainly due to electromagnetic torque and the torque connected with gravitational wave radiation. For the latter, first, a magnetic-field-caused equatorial ellipticity can generate gravitational-quadrupole radiation, which however cannot provide an essential contribution to the spin-down unless with an extremely high magnetic field (Usov 1992). Second, a relatively stronger gravitational wave radiation can be produced through some nonaxisymmetric stellar perturbations. For nascent neutron stars, such perturbations can easily be created by $r$-mode instability, which arises from the action of the Coriolis force with positive feedback (Andersson 1998; Friedman \& Morsink 1998) succumbing to gravitational radiation-driven Chandrasekhar-Friedman-Schutz instability.
Following a phenomenological second-order model for the $r$-mode evolution (Owen et al. 1998; Sá 2004), we can calculate the spin evolution of a magnetar by (Sá 2004; Yu et al. 2009a)

$$
\frac{d P}{d t}=\frac{4 \bar{\alpha}^{2}}{15}(\delta+2) \frac{P}{\tau_{g}}+\frac{P}{\tau_{m}},
$$

where $P$ is the spin period of the magnetar, $\bar{\alpha}$ is the dimensionless amplitude of the $r$-modes, and $\delta$ is a free parameter describing the initial degree of the differential rotation of the star. The gravitational and magnetic braking timescales can be written as $\tau_{g}=144 P_{-3}^{6}$ s and $\tau_{m}=4 \times 10^{5} B_{14}^{-2} P_{-3}^{2}$ s, respectively. ${ }^{3}$ The viscous damping of the $r$-modes is ignored here due to the high temperatures of $\sim 10^{10} \mathrm{~K}$ in nascent neutron stars. Correspondingly, the evolution of the $r$-mode amplitude can be calculated from (Sá 2004; Yu et al. 2009a)

$$
\frac{d \bar{\alpha}}{d t}=\left[1+\frac{2 \bar{\alpha}^{2}}{15}(\delta+2)\right] \frac{\bar{\alpha}}{\tau_{g}}+\frac{\bar{\alpha}}{2 \tau_{m}} .
$$

In the case of $\tau_{g, i} \gg \tau_{m, i}$ (i.e., $B \gg B_{c}=5 \times 10^{15} P_{i,-3}^{-2} \mathrm{G}$ ), the spin-down would be dominated exclusively by the magnetic dipole radiation, and the $r$-modes cannot arise sufficiently rapidly. In this case, the spin evolution can easily be expressed as usual as $P(t)=P_{i}\left(1+t / T_{m}\right)^{1 / 2}$ with $T_{m}=2 \times 10^{5} B_{14}^{-2} P_{i,-3}^{2} \mathrm{~s}$.

In contrast, for not very high magnetic fields (i.e., $\tau_{g, i} \ll$ $\left.\tau_{m, i}\right)$, the spin-down should be first dominated by the gravitational wave radiation. By ignoring the magnetic term, Equations (1) and (2) can be solved analytically, and an asymptotic solution can be written as (Sá \& Tomé 2005, 2006)

$$
P(t) \approx \begin{cases}P_{i}\left[1-\frac{2}{15} \bar{\alpha}_{i}^{2}(\delta+2) \exp \left(2 t / \tau_{g, i}\right)\right]^{-1}, & \text { for } t<T_{g}, \\ 1.6 P_{i}\left(t / \tau_{g, i}\right)^{1 / 5}, & \text { for } t>T_{g}\end{cases}
$$

Here, the break time can be solved by $\left(d^{2} \bar{\alpha} / d t^{2}\right)_{t=T g}=0$ to be $T_{g}=a \times 10^{3} P_{i,-3}^{6} \mathrm{~s}$, where the prefactor $0.7<a<3.2$ for a wide parameter region of $10^{-10}<\bar{\alpha}_{i}<10^{-6}$ and $0<\delta<10^{8}$ (Yu et al. 2009b). As the spin period increases, the magnetic braking effect would eventually exceed the gravitational braking effect and then the time dependence of the spin period changes from $P \propto t^{1 / 5}$ to $P \propto t^{1 / 2}$. We denote the change time by $T_{c}$, which will be given explicitly later.

\section{GRB X-RAY AFTERGLOW EMISSION}

After the bursts, the central remanent magnetars could still keep abundant rotational energy, although a considerable fraction has been expanded on the bursts. This remaining energy could be released peacefully and persistently, and drive a continuous magnetar winds. We believe that the magnetar winds are probably able to produce long-lasting emission (i.e., afterglow emission) through some internal dissipation mechanisms such as magnetic reconnection (e.g., Giannios \& Spruit 2005), the terminative shock of the winds (e.g., Dai 2004; Yu \& Dai 2007), etc. Such an internal-origin emission component would of course compete with the external shock emission component, while the latter alone is usually challenged by some multi-wavelength afterglow observations (e.g., chromatic LC breaks).

\footnotetext{
3 Hereafter, some basic structural parameters such as the mass, the radius, and the moment of inertial of the magnetar are taken to be $1.4 M_{\odot}, 10^{6} \mathrm{~cm}$, and $10^{45} \mathrm{~g} \mathrm{~cm}^{2}$, respectively. Additionally, the convention $Q_{x}=Q / 10^{x}$ is adopted in cgs units, and a subscript " $i$ " represents the initial values of the quantities.
} 
In order to compare with X-ray observations, we first simply assume that the (isotropically equivalent) luminosity of the wind $\mathrm{X}$-ray afterglow emission tracks the magnetic dipole luminosity of the magnetars with a constant fraction $(\xi ; \mathrm{X}$-ray radiation efficiency) as

$$
\mathcal{L}_{X}^{\mathrm{mw}}=\xi L_{\mathrm{md}} / f_{B}=10^{47} \xi_{-1} f_{B,-1}^{-1} L_{\mathrm{md}, 47} \mathrm{erg} \mathrm{s}^{-1},
$$

where $f_{B}=\left(1-\cos \theta_{w}\right)$ is the beaming factor of the magnetar winds with $\theta_{w}$ being the half-opening angle of the winds. To be specific, the magnetic dipole luminosity of a magnetar can be calculated by

$$
L_{\mathrm{md}}(t)=\frac{I \Omega^{2}}{\tau_{m}}=10^{47} F(t) B_{14}^{2} P_{i,-3}^{-4} \mathrm{erg} \mathrm{s}^{-1},
$$

where $I$ is the moment of inertia of the star and $\Omega=2 \pi / P$ is the spin frequency. According to the analysis on the spin-down of the magnetars, the time dependence $F(t)$ of the magnetic dipole luminosity can be expressed approximately as

for $B>B_{c}$,

$$
F(t) \approx \begin{cases}t^{0}, & t<T_{m} \\ \left(\frac{t}{T_{m}}\right)^{-2}, & t>T_{m}\end{cases}
$$

for $B<B_{c}$,

$$
F(t) \approx \begin{cases}t^{0}, & t<T_{g}, \\ \left(\frac{t}{T_{g}}\right)^{-q}, & T_{g}<t<T_{c}, \\ \left(\frac{T_{c}}{T_{g}}\right)^{-q}\left(\frac{t}{T_{c}}\right)^{-2}, & t>T_{c},\end{cases}
$$

where $T_{c}=\left(T_{m}^{2} / T_{g}^{q}\right)^{1 /(2-q)}$. Following Equation (3), the value of $q$ can be taken as 0.8 approximately (a more exact numerical calculation would show $q \sim 1$ ). As shown by Equation (7) for $B<B_{c}$, we surprisingly find that the double-broken power-law behavior of $\mathcal{L}_{X}^{\mathrm{mw}}$ is in good agreement with the so-called canonical X-ray LC including all of the shallow decay, normal decay, and steeper-than-normal decay phases. This may be just a coincidence, but alternatively it also seems acceptable to ascribe some observed double-broken power-law $\mathrm{X}$-ray afterglow emission to the magnetar winds rather than the external shocks. In this case, the change in the slopes of the LCs is just because of the evolution of the magnetic dipole luminosity, but independent of the external shock physics. As an analogical consideration for the magnetars with $B>B_{c}$, Equation (6) also predicts some X-ray LCs behaving as a plateau followed by a steep decay with $\alpha \sim 2$. However, the rare observation of such type of X-ray LCs implies that the magnetic field strengths of the GRB magnetars are generally lower than the critical value $B_{c}{ }^{4}$ So, we would mainly be concerned with the case of $B<B_{c}$ in the following calculations.

Although the internal X-ray afterglow emission is suggested above, we still think that the external shock could play an important role in the afterglow emission. In the calculation of shock dynamics, we also take into account the energy injection into the shock from the wind as considered before (e.g., Dai \& Lu 1998a, 1998b; Zhang \& Mészáros 2001), even though a part of the wind energy could have been radiated directly. Following Equations (5) and (7) for $B<B_{c}$, the increasing (isotropically

\footnotetext{
4 We will test this argument in Section 4 and give some related discussion in
} Section 5 . equivalent) shock energy can be approximately written as (for $q \leqslant 1)$

$$
\mathcal{E}(t) \approx \mathcal{E}_{i} \times \begin{cases}t^{0}, & t<T_{\mathrm{ei}}, \\ \left(\frac{t}{T_{\mathrm{ei}}}\right), & T_{\mathrm{ei}}<t<T_{g}, \\ \left(\frac{T_{g}}{T_{\mathrm{ei}}}\right)\left(\frac{t}{T_{g}}\right)^{1-q}, & T_{g}<t<T_{c}, \\ \left(\frac{T_{g}}{T_{\mathrm{ei}}}\right)\left(\frac{T_{c}}{T_{g}}\right)^{1-q} \equiv \mathcal{E}_{f}, & t>T_{c} .\end{cases}
$$

The starting time of the energy increase can be calculated by $T_{\text {ei }}=\mathcal{E}_{i} /\left(\zeta f_{B}^{-1} L_{\mathrm{md}, \mathrm{i}}\right)=100 \zeta_{0}^{-1} \mathcal{E}_{i, 50} f_{B,-1} L_{\mathrm{md}, \mathrm{i}, 47}^{-1} \mathrm{~s}$, where $\zeta \leqslant(1-\xi)$ is the fraction of the wind energy that is injected into the shock. Equation (8) shows that the energy injection process can be divided into two stages for $q<1$. However, since the value of $q$ is actually very close to 1 , the energy increase of the shock after $T_{g}$ is difficult to be detected and thus can be neglected. Therefore, in comparison with the conventional energy injection model proposed by Dai \& Lu (1998a, 1998b) and Zhang \& Mészáros (2001), the main difference here is that the duration of the energy injection is determined by $T_{g}$ rather than $T_{m}$. For shock dynamic evolution, ${ }^{5}$ we first introduce the deceleration time of the shock as $T_{\mathrm{dec}}=3 \mathcal{E}_{i, 50}^{1 / 3} \Gamma_{i, 2.3}^{-8 / 3} n_{0}^{-1 / 3} \mathrm{~s}$ $\left(<T_{\mathrm{ei}}<T_{g}\right.$ ). Before $T_{\mathrm{dec}}$, the shock deceleration can be neglected (i.e., $\Gamma \sim \Gamma_{i}$ ) since $\mathcal{M}_{\mathrm{sw}}$ is insufficiently high. After $T_{\mathrm{dec}}$, the dynamic evolution of the shock can be obtained from the energy conservation law $\mathcal{E}=\Gamma^{2} \mathcal{M}_{\mathrm{sw}} c^{2}$ as

$$
\Gamma(t)=\left(\frac{3 \mathcal{E}}{32 \pi n m_{p} c^{5} t^{3}}\right)^{1 / 8} \propto \begin{cases}t^{-3 / 8}, & T_{\mathrm{dec}}<t<T_{\mathrm{ei}}, \\ t^{-1 / 4}, & T_{\mathrm{ei}}<t<T_{g}, \\ t^{-3 / 8}, & t>T_{g} .\end{cases}
$$

Obviously, the shock deceleration during $T_{\mathrm{ei}}<t<T_{g}$ is effectively decreased by the energy injection.

For the afterglow emission of interest, here we only concern the shock emission after $T_{\mathrm{ei}}$. Using the above dynamic results and following Sari et al. (1998), the (isotropically equivalent) luminosity of the shock synchrotron X-ray afterglows can be given analytically by

$\mathcal{L}_{X}^{\mathrm{sh}}=\mathcal{L}_{X, \mathrm{ei}}^{\mathrm{sh}} \times \begin{cases}\left(\frac{t}{T_{\mathrm{ei}}}\right)^{-(p-2) / 2}, & T_{\mathrm{ei}}<t<T_{g}, \\ \left(\frac{T_{g}}{T_{\mathrm{ei}}}\right)^{-(p-2) / 2}\left(\frac{t}{T_{g}}\right)^{-(3 p-2) / 4}, & t>T_{g},\end{cases}$

where $p$ is the spectral index of the energy distribution of the shock-accelerated electrons. The luminosity at $T_{\text {ei }}$ reads

$$
\begin{aligned}
\mathcal{L}_{X, \mathrm{ei}}^{\mathrm{sh}} \approx & 10^{46}\left(g_{p,-0.5} \epsilon_{e,-1}\right)^{p-1}\left(\zeta_{0} f_{B,-1}^{-1} L_{\mathrm{md}, \mathrm{i}, 47}\right)^{(3 p-2) / 4} \\
& \times\left(v_{X, 17.5} \mathcal{E}_{i, 50}\right)^{(2-p) / 2} \epsilon_{B,-2}^{(p-2) / 4} \mathrm{erg} \mathrm{s}^{-1}
\end{aligned}
$$

where $g_{p}=(p-2) /(p-1), v_{X}$ is the X-ray frequency, and $\epsilon_{e}$ and $\epsilon_{B}$ are the equipartition factors of the electron internal energy and magnetic energy, respectively.

Equation (11) shows that, for $p$ being not much higher than 2 , the shock luminosity is mainly sensitive to the parameters $\epsilon_{e}$,

\footnotetext{
5 Here, some shock-related quantities are defined as follows: $\Gamma$ and $R=2 \Gamma^{2} c t$ are the Lorentz factor and the radius of the shock,

$\mathcal{M}_{\mathrm{sw}}=\frac{4}{3} \pi R^{3} \mathrm{~nm}_{p}$ is the (isotropically equivalent) mass of the medium swept up by the shock, and $n$ is the number density of the circum medium. $m_{p}$ and $c$ are the mass of proton and the speed of light.
} 


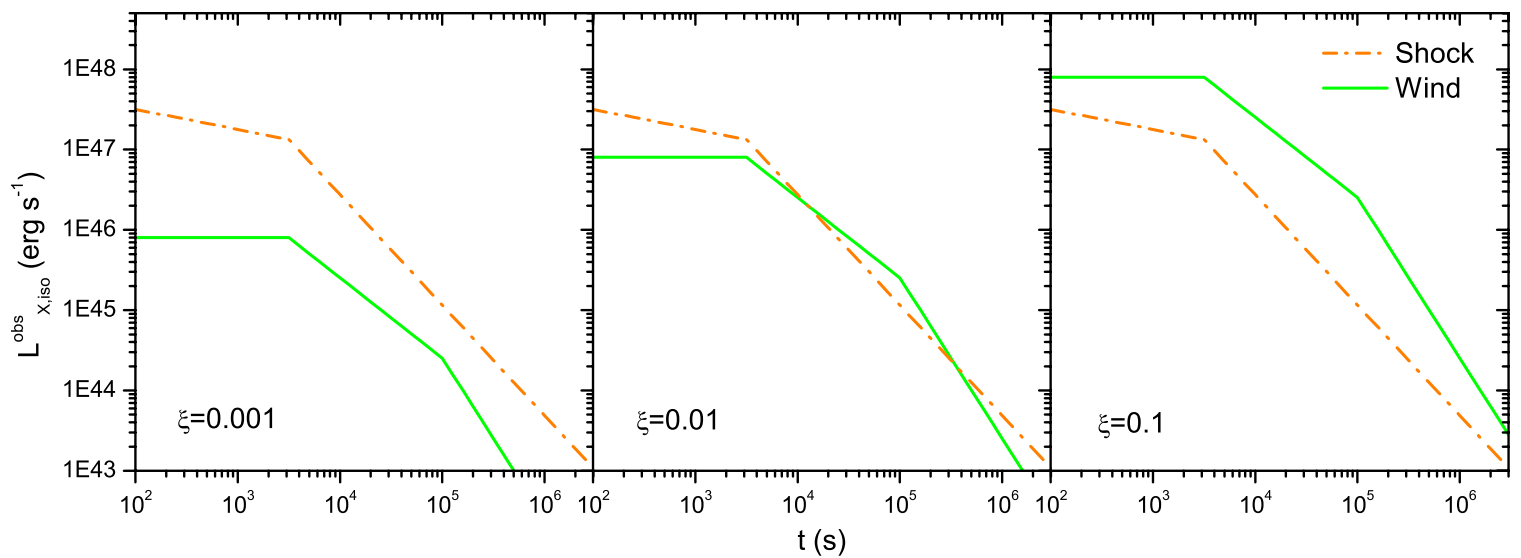

Figure 1. Illustrative X-ray $\left(v_{X}=3 \times 10^{17} \mathrm{~Hz}\right)$ afterglow LCs contributed by the magnetar wind with varying $\xi$ as labeled and by the energized shock with $\epsilon_{e}=0.2$ and $g_{p,-0.5}=\epsilon_{B,-2}=E_{i, 50}=1$. The magnetar parameters are taken to be $P_{i}=1.2 \mathrm{~ms}, B=4 \times 10^{14} \mathrm{G}$, and $f_{B}=0.1$.

(A color version of this figure is available in the online journal.)

$\zeta, L_{\mathrm{md}}$, and $f_{B}$. So, an upper limit for the shock luminosity for $t>T_{\mathrm{dec}}$ can be given as follows:

$$
\mathcal{L}_{X, \text { upper }}^{\text {sh }}=\epsilon_{e} \zeta L_{\mathrm{md}, \mathrm{i}} / f_{B}=10^{47} \epsilon_{e,-1} \zeta_{0} f_{B,-1}^{-1} L_{\mathrm{md}, \mathrm{i}, 47} \mathrm{erg} \mathrm{s}^{-1} .
$$

A comparison between Equations (4) and (12) shows that the shock emission can exceed the wind emission only for $\epsilon_{e} \gg \xi /(1-\xi)$. By varying the value of $\xi$ and fixing $\epsilon_{e}=0.2$, we plot the wind- and shock-contributed X-ray LCs in Figure 1. Combining the two emission components, the model in principle predicts three types of LCs as exhibited, i.e., an energized shock-dominated type, a magnetar wind-dominated type, and an intermediate type. In particular, the intermediate type LCs could have an interesting but complicated profile. It will be a valuable attempt to find some observational cases with such a profile, although it would not be easy. For a general investigation, we would only be concerned with the other two types in this paper. It seems somewhat too simple that nearly all of the Swift-XRT afterglows can be summarized by a canonical LC. This view obscures the possible diversity of the physical origins of the $\mathrm{X}$-ray afterglows. In our opinion, we had better treat all of the observed afterglows as a collective of several different classes, which corresponds to different afterglow mechanisms.

\section{CONFRONTING THE MODEL WITH OBSERVATIONS}

\subsection{Fittings to Some Observational LCs}

As shown in Figure 1, both the energized shock- and magnetar wind-dominated afterglow LCs initially have a very flat segment, the decay index of which is close to zero if $p$ is not much higher than 2 . Therefore, we select observational samples from the public XRT-team LC repository (Evans et al. 2007, 2009) between 2005 January and 2009 December under four criteria: (1) the data are rich enough to clearly exhibit the profile of the LC, and no flare appears after the initial steep decay phase; (2) a remarkable plateau emission $(\alpha<0.3)$ immediately follows the initial steep decay phase; (3) the decay index of the last segment of the LC is not much larger than 2; and (4) the GRB's redshift is known. As a result, 16 representative samples are obtained, which naturally fall into two classes as shown in Figures 2 and 3. The former LCs have only one break after the initial steep decay phase, while the latter LCs have two breaks. Using the following smoothed broken and doublebroken power-law functions ( $w=3$; Liang et al. 2007),

$$
F_{X}(t)=F_{X, b}^{(1)}\left[\left(\frac{t}{T_{b}^{(1)}}\right)^{w \alpha_{1}^{(1)}}+\left(\frac{t}{T_{b}^{(1)}}\right)^{w \alpha_{2}^{(1)}}\right]^{-1 / w}
$$

and

$$
\begin{aligned}
F_{X}(t)= & F_{X, b}^{(2)}\left[\left(\frac{t}{T_{b 1}^{(2)}}\right)^{w \alpha_{1}^{(2)}}+\left(\frac{t}{T_{b 1}^{(2)}}\right)^{w \alpha_{2}^{(2)}}\right. \\
& \left.+\left(\frac{T_{b 2}^{(2)}}{T_{b 1}^{(2)}}\right)^{w \alpha_{2}^{(2)}}\left(\frac{t}{T_{b 2}^{(2)}}\right)^{w \alpha_{3}^{(2)}}\right]^{-1 / w},
\end{aligned}
$$

to fit the selected one-break and two-break LCs, respectively, we can obtain the slopes $(\alpha)$, the break times $\left(T_{b}\right)$, and the $\mathrm{X}$-ray fluxes at the first break $\left(F_{X, b}\right)$ of the LCs, as listed in Tables 1 and 2. The distributions of these fitting parameters are exhibited in Figure 4 (solid histogram), which shows that (1) $\alpha_{1}^{(1)}$ is statistically a bit larger than $\alpha_{1}^{(2)}$, and (2) $\alpha_{2}^{(1)}$ could be usually larger than $\sim 1.2-1.4$, whereas $\alpha_{2}^{(2)}$ is inclined to be smaller than $\sim 1.2-1.4$, as expected by our model. But when we use Equation (13) to fit only the first two segments of the two-break LCs, the difference between $\alpha_{2}^{(1)}$ and $\alpha_{2}^{(2)}$ becomes ambiguous as shown by the dashed open histogram in Figure 4. In contrast to the centralization of $T_{b 2}^{(2)}$ at $\sim 10^{4-5} \mathrm{~s}$, the obvious lack at least before $\sim 10^{6} \mathrm{~s}$ of the second break in the one-break LCs still suggests a clear difference between these two types of LCs, even though a longer term $\left(>10^{6} \mathrm{~s}\right)$ observation may be able to detect a second break in some so-called one-break afterglows.

For the above observational results, a plausible explanation can in principle be provided by our model where the one- and two-break afterglows are respectively attributed to the shock and wind emission, although it seems hard to observationally judge (within the present small sample) whether there is an intrinsic difference between the one- and two-break LCs. On the one hand, for the temporal decay indices, the values of $\alpha_{1,2}^{(1)}$ and $\alpha_{1,2,3}^{(2)}$ are statistically in rough agreement with the model-predicted $\alpha_{1,2}^{(\mathrm{sh})}$ and $\alpha_{1,2,3}^{(\mathrm{mw})}$, respectively. On the other hand, the similar 


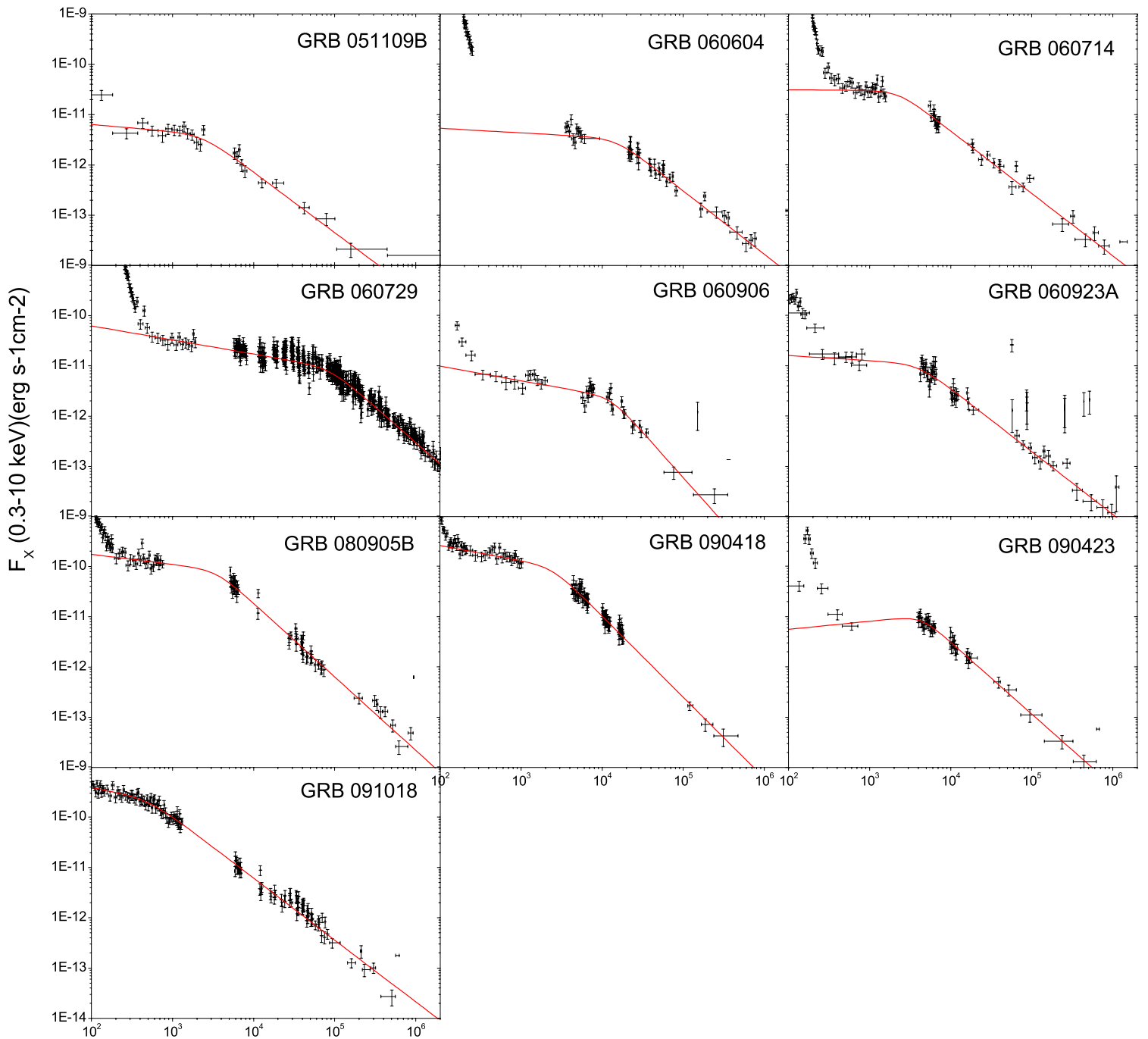

$t(s)$

Figure 2. Selected one-break X-ray afterglow LCs, which can be fitted by Equation (13) as shown by the solid lines. (A color version of this figure is available in the online journal.)

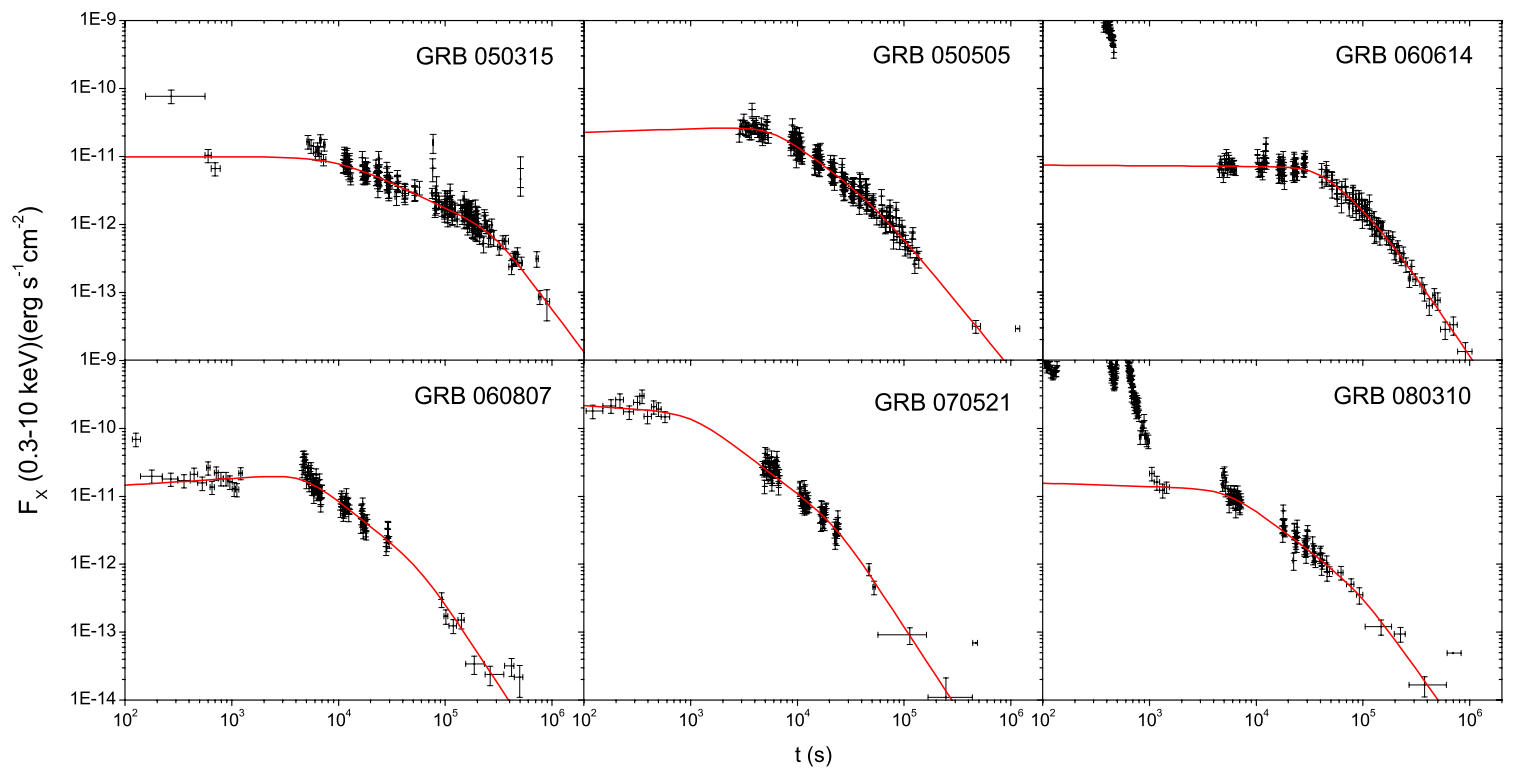

Figure 3. Selected two-break X-ray afterglow LCs, which can be fitted by Equation (14) as shown by the solid lines. (A color version of this figure is available in the online journal.) 

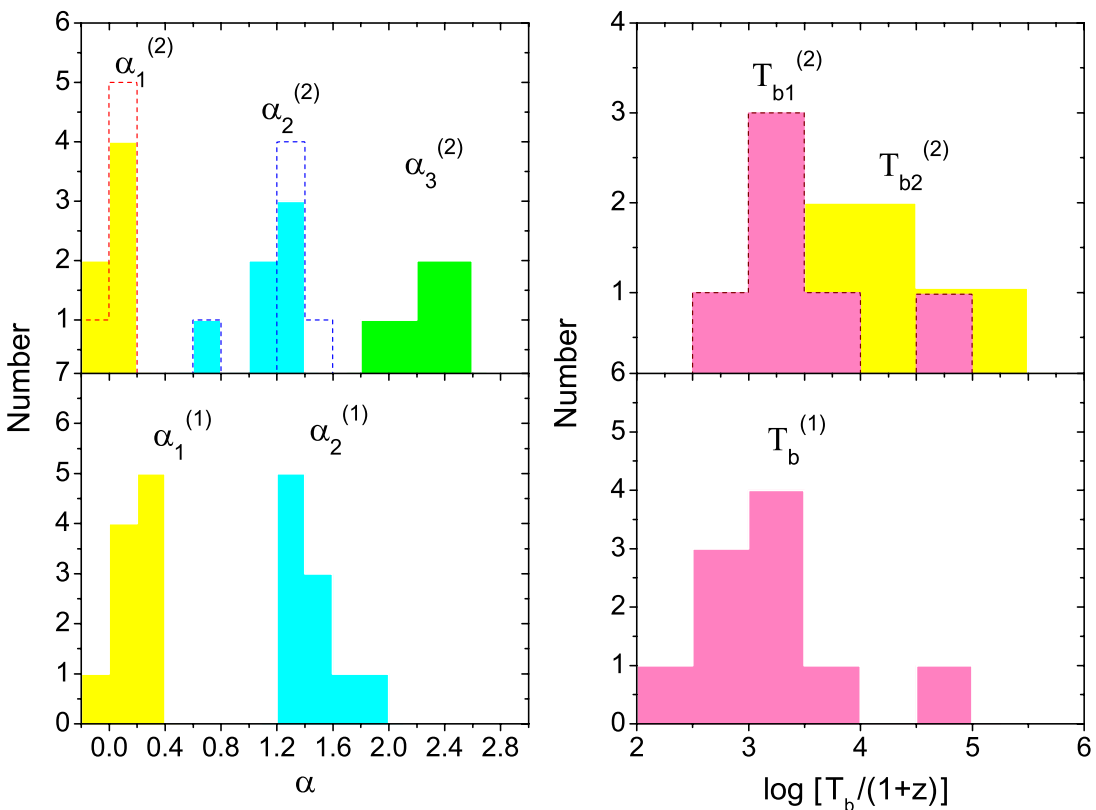

Figure 4. Distributions of the fitting parameters of the one-break (lower panel) and two-break (upper panel) X-ray afterglow LCs (solid histogram), which are respectively fitted by Equations (13) and (14). The dashed open histogram shows the results of the fitting to the first two segments of the LCs by Equation (13). (A color version of this figure is available in the online journal.)

Table 1

Fitting Parameters of the One-break X-ray Afterglow LCs and the Corresponding Magnetar Parameters

\begin{tabular}{llrlrccc}
\hline \hline \multicolumn{1}{c}{ GRB } & $z^{\mathrm{a}}$ & \multicolumn{1}{c}{$\alpha_{1}^{(1)}$} & $\alpha_{2}^{(1)}$ & $\begin{array}{c}T_{b}^{(1)} \\
\left(10^{3} \mathrm{~s}\right)\end{array}$ & $\begin{array}{c}F_{X, b}^{(1)} \\
\left(10^{-11} \mathrm{erg} \mathrm{s}^{-1} \mathrm{~cm}^{-1}\right)\end{array}$ & $\begin{array}{c}P_{i}^{\mathrm{b}} \\
\left(10^{-3} \mathrm{~s}\right)\end{array}$ & $\begin{array}{c}B^{\mathrm{b}} \\
\left(10^{14} \mathrm{G}\right)\end{array}$ \\
\hline $051109 \mathrm{~B}$ & 0.08 & 0.14 & 1.20 & 2.34 & 0.41 & 1.14 & 0.20 \\
060604 & 2.68 & 0.09 & 1.26 & 14.68 & 0.34 & 1.26 & 4.68 \\
060714 & 2.711 & 0.01 & 1.24 & 2.23 & 3.01 & 0.92 & 5.55 \\
060729 & 0.54 & 0.28 & 1.42 & 88.33 & 0.93 & 1.96 & 3.87 \\
060906 & 3.686 & 0.29 & 1.80 & 12.83 & 0.24 & 1.18 & 4.81 \\
$060923 \mathrm{~A}$ & $<2.8$ & 0.10 & 1.25 & 4.00 & 1.10 & 1.00 & 4.78 \\
$080905 \mathrm{~B}$ & 2.374 & 0.20 & 1.46 & 3.49 & 8.52 & 1.00 & 8.64 \\
090418 & 1.608 & 0.30 & 1.61 & 2.43 & 9.91 & 0.99 & 6.21 \\
090423 & 8.26 & -0.16 & 1.43 & 4.35 & 1.02 & 0.88 & 9.00 \\
091018 & 0.971 & 0.29 & 1.23 & 0.50 & 24.35 & 0.80 & 3.53 \\
\hline
\end{tabular}

Notes.

a The GRB redshifts are taken from the Web site http://www.mpe.mpg.de/ jcg/grbgen.html.

b The values here are obtained with model parameters $v_{X, 17.5}=g_{p,-0.5}=\epsilon_{e,-1}=\epsilon_{B,-2}=\mathcal{E}_{i, 50}=$ $f_{B,-1}=1$ and $\zeta \sim 1$.

Table 2

Fitting Parameters ${ }^{\mathrm{a}}$ of the Two-break X-ray Afterglow LCs and the Corresponding Magnetar Parameters

\begin{tabular}{|c|c|c|c|c|c|c|c|c|c|c|}
\hline GRB & $z^{\mathrm{b}}$ & $\alpha_{1}^{(2)}$ & $\alpha_{2}^{(2)}$ & $\alpha_{3}^{(2)}$ & $\begin{array}{c}T_{b 1}^{(2)} \\
\left(10^{3} \mathrm{~s}\right) \\
\end{array}$ & $\begin{array}{c}T_{b 2}^{(2)} \\
\left(10^{5} \mathrm{~s}\right) \\
\end{array}$ & $\begin{array}{c}F_{X, b}^{(2)} \\
\left(10^{-11} \mathrm{erg} \mathrm{s}^{-1} \mathrm{~cm}^{-1}\right)\end{array}$ & $\begin{array}{c}P_{i}^{\mathrm{c}} \\
\left(10^{-3} \mathrm{~s}\right)\end{array}$ & $\begin{array}{c}B^{\mathrm{c}} \\
\left(10^{14} \mathrm{G}\right) \\
\end{array}$ & $\xi / f_{B,-1}$ \\
\hline 050315 & 1.949 & $0.00(0.00)$ & $0.74(0.77)$ & 2.11 & $9.63(10.35)$ & 2.82 & $0.99(0.98)$ & 1.22 & 4.06 & 0.035 \\
\hline 050505 & 4.27 & $-0.05(0.01)$ & $1.14(1.26)$ & 1.92 & $5.60(6.64)$ & 0.52 & $2.77(2.48)$ & 1.01 & 7.86 & 0.082 \\
\hline 060614 & 0.125 & $0.01(0.02)$ & $1.40(1.59)$ & 2.32 & $37.00(38.90)$ & 1.42 & $0.70(0.68)$ & 1.79 & 3.12 & 0.0003 \\
\hline 060807 & $<3.4$ & $-0.10(-0.10)$ & $1.24(1.25)$ & 2.40 & $4.74(4.74)$ & 0.59 & $2.14(2.13)$ & 1.01 & 7.27 & 0.044 \\
\hline 070521 & 1.35 & $0.11(0.12)$ & $1.20(1.32)$ & 2.40 & $1.03(1.26)$ & 0.23 & $16.86(15.94)$ & 0.87 & 8.48 & 0.014 \\
\hline 080310 & 2.42 & $0.05(0.12)$ & $1.19(1.25)$ & 2.22 & $5.41(6.34)$ & 0.92 & $1.28(1.15)$ & 1.08 & 5.92 & 0.022 \\
\hline
\end{tabular}

Notes.

a The numbers in the brackets are obtained by fitting the first two segments by Equation (13).

b The GRB redshifts are taken from the Web site http://www.mpe.mpg.de/ jcg/grbgen.html.

c The values are inferred with $a \sim 1$.

distributions of $T_{b}^{(1)}$ and $T_{b 1}^{(2)}$ can also be naturally understood by ascribing both of them to a same physical origin, i.e., the $r$-mode instability requiring $T_{b}^{(1)} \sim T_{b 1}^{(2)} \sim(1+z) T_{g}$. Of course, for a more comprehensive observational test, an investigation into the afterglow spectral information is desired. On the theoretical aspect, it would be useful to derive some closure 


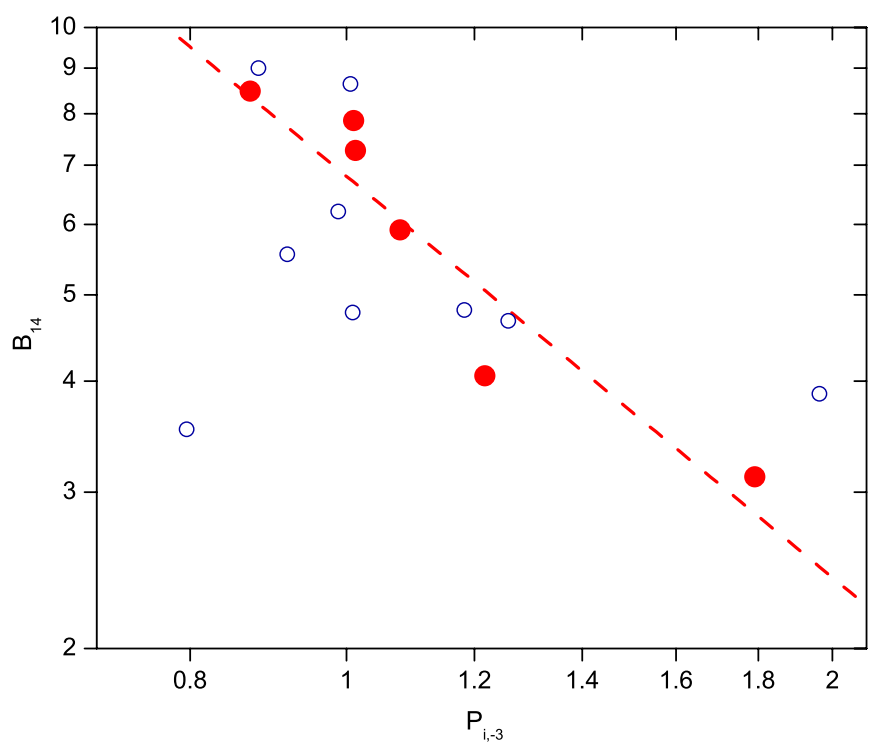

Figure 5. Distribution in the $P_{i}-B$ panel of the magnetars inferred from both the one-break (open cycle) and two-break (solid cycle) X-ray afterglows. The dashed line represents $B_{14} \sim 7 P_{i,-3}^{-3 / 2}$.

(A color version of this figure is available in the online journal.)

relations between the temporal decay index and the spectral index in the wind emission model, as done by some authors for the shock model (Zhang et al. 2006; Liang et al. 2007; Willingale et al. 2007). Unfortunately, such an attempt now is restricted by the ignorance of the specific radiation mechanisms of the winds.

\subsection{Implications for the Central Magnetars}

Following the above comparison between the model and the observations, here we simply connect the selected one- and twobreak afterglows with the shock- and wind-dominated emission, respectively, in order to give insight into the properties of the possible existing central magnetars. To be specific, for the twobreak afterglows, the values of the characteristic timescales in the model can easily be determined by $T_{g} \sim T_{b 1}^{(2)} /(1+z)$, $T_{c} \sim T_{b 2}^{(2)} /(1+z)$, and $T_{m}=\left(T_{g} T_{c}\right)^{1 / 2}$ (for $q=1$ ). Then the spin periods and magnetic field strengths of the magnetars can easily be obtained by

$$
\begin{gathered}
P_{i,-3}=a^{-1 / 6} T_{g, 3}^{1 / 6}, \\
B_{14}=1.4 P_{i,-3} T_{m, 5}^{-1 / 2} .
\end{gathered}
$$

However, for the one-break afterglows, the values of $T_{c}$ and thus $T_{m}$ cannot be found from the observations. So, we have to seek help from Equations (5), (11), and $\mathcal{L}_{X, \mathrm{ei}}^{\mathrm{sh}} \sim \mathcal{L}_{X, b}^{\mathrm{obs}}{ }^{6}$ Then we obtain

$$
B_{14} \approx\left[\left(\mathcal{L}_{X, b, 46}^{\mathrm{obs}}\right)^{4 /(3 p-2)} P_{i,-3}^{2}\right]^{1 / 2}
$$

with model parameters $v_{X, 17.5}=g_{p,-0.5}=\epsilon_{e,-1}=\epsilon_{B,-2}=$ $\mathcal{E}_{i, 50}=f_{B,-1}=1$ and $\zeta \sim 1$. Using the above equations, we derive the values of the magnetar parameters $P_{i}$ and $B$ from

\footnotetext{
6 The isotropically equivalent observational X-ray luminosity at $T_{b}^{(1)}$ can be calculated by $\mathcal{L}_{X, b}^{\mathrm{obs}}=4 \pi d_{l}^{2} F_{X, b}^{(1)}$, where the luminosity distance reads $d_{l}(z)=\frac{(1+z) c}{H_{0}} \int_{0}^{z}\left[\Omega_{\Lambda}+\Omega_{m}\left(1+z^{\prime}\right)^{3}\right]^{-1 / 2} d z^{\prime}$ with cosmological parameters $\Omega_{\Lambda}=0.73, \Omega_{m}=0.27$, and $H_{0}=73 \mathrm{~km} \mathrm{~s}^{-1} \mathrm{Mpc}^{-1}$.
}

both the one-break and two-break samples, as listed in Tables 1 and 2, respectively. Figure 5 further shows that, for the model parameters adopted, there is no clear separation between the two samples. However, keep in mind that the magnetar parameters inferred from the one-break afterglows are strongly sensitive to the uncertain model parameters (especially $p, \epsilon_{e}$, and $f_{B}$ ).

As a conservative treatment, here we only analyze the relatively credible magnetar parameters from the two-break afterglows. First, the hypothesis of $B<B_{c}$ is favored by the inferred magnetic field strengths as $B \sim 10^{14-15} \mathrm{G}$. Second, by calculating the magnetic dipole luminosity using Equation (5), we can estimate the X-ray radiation efficiency of the magnetar winds, which reads

$$
\xi=f_{B} \mathcal{L}_{X, b}^{\mathrm{obs}} / L_{\mathrm{md}, \mathrm{i}} \sim(0.01-0.1) f_{B,-1},
$$

as shown in Table 2. Finally, we even find that the magnetic field strengths and the spin periods of the magnetars may satisfy a loose correlation as $B \propto P_{i}^{-3 / 2}$. This is qualitatively in agreement with the dynamo origin of the magnetic fields (e.g., $\mathrm{Xu}$ et al. 2002).

\section{SUMMARY AND DISCUSSION}

Based on two assumptions that (1) some GRB central objects are millisecond magnetars and (2) the magnetar winds can continuously produce X-ray emission whose luminosity tracks the magnetic dipole luminosity, we investigate the temporal behaviors of the GRB X-ray afterglows arising from an emitting magnetar wind and an energized external shock together. The competition between the internal- and external-origin emission components determines the diversity of the observed X-ray afterglow LCs. A comparison between the model and observations shows that the model-predicted shock- and wind-dominated emission is qualitatively consistent with the observed one-break and two-break afterglows, respectively.

In the conventional shock model, the second break of the two-break afterglows is always connected to the jet break, which is, however, seriously challenged by the usually observed chromatic breaks (Liang et al. 2008). In contrast, the chromatic breaks could be acceptable for the internal-origin emission. On the one hand, such an argument is supported by the lack of the optical counterparts of X-ray flares, which are of internal origin. On the other hand, in view of the possible small radii where the internal dissipations occur, the low-frequency emission of the wind is quite likely to be suppressed, for example, by some selfabsorption effects. Therefore, for some GRBs, while their X-ray afterglows are contributed by the magnetar winds, the optical emission could be still dominated by the external shocks. In this case, chromatic breaks would be detected naturally.

In this paper, we are mainly concerned with the ordinary GRB $\mathrm{X}$-ray afterglows that may be associated with a magnetar with a relatively low magnetic field ( $B<B_{c}$ ). For simplicity, we do not pay much attention to some unusual X-ray afterglows such as the afterglows behaving as a plateau followed by a steep decay (e.g., GRBs 060607A and 070110). As discussed in Section 3, such X-ray afterglows could be internal afterglows produced by the magnetars with relatively high magnetic fields $\left(B>B_{c}\right)$. In this case, however, if a low value of $B$ is found, the related magnetar could be a candidate of strange quark stars rather than neutron stars, since only strange stars can suppress the $r$-mode instability effectively (Yu et al. 2009b).

This work made use of data supplied by the UK Swift Science Data Centre at the University of Leicester. We acknowledge 
useful comments by the referee. This work is supported by the GRF Grants of the Government of the Hong Kong SAR under HKU7011/09P. Y.W.Y. is also supported by the SelfDetermined Research Funds of CCNU (CCNU09A01020) from the colleges' basic research and operation of MOE.

\section{REFERENCES}

Andersson, N. 1998, ApJ, 502, 708

Bucciantini, N., et al. 2009, MNRAS, 396, 2038

Cannizzo, J. K., \& Gehrels, N. 2009, ApJ, 700, 1047

Corsi, A., \& Mészáros, P. 2009, ApJ, 702, 1171

Dai, Z. G. 2004, ApJ, 606, 1000

Dai, Z. G., \& Lu, T. 1998a, Phys. Rev. Lett., 81, 4301

Dai, Z. G., \& Lu, T. 1998b, A\&A, 333, L87

Dai, Z. G., Wang, X. Y., Wu, X. F., \& Zhang, B. 2006, Science, 311, 1127

De Pasquale, M., et al. 2007, MNRAS, 377, 1638

Drenkhahn, G. 2002, A\&A, 387, 714

Drenkhahn, G., \& Spruit, H. C. 2002, A\&A, 391, 1141

Duncan, R. C., \& Thompson, C. 1992, ApJ, 392, L9

Evans, P. A., et al. 2007, A\&A, 469, 379

Evans, P. A., et al. 2009, MNRAS, 397, 1177

Fan, Y. Z., \& Xu, D. 2006, MNRAS, 372, L19

Fenimore, E. E., Madras, C. D., \& Nayakshin, S. 1996, ApJ, 473, 998

Friedman, J. L., \& Morsink, S. M. 1998, ApJ, 502, 714

Gehrels, N., et al. 2004, ApJ, 611, 1005

Ghisellini, G., Ghirlanda, G., Nava, L., \& Firmani, C. 2007, ApJ, 658, L75

Giannios, D., \& Spruit, H. C. 2005, A\&A, 430, 1

Kluźniak, W. 1998, ApJ, 509, L37

Kluźniak, W., \& Ruderman, M. 1998, ApJ, 505, L113

Kumar, P., Narayan, R., \& Johnson, J. L. 2008, Science, 321, 376

Kumar, P., \& Panaitescu, A. 2000, ApJ, 541, L51
Liang, E.-W., Racusin, J. L., Zhang, B., Zhang, B.-B., \& Burrows, D. N 2008, ApJ, 675, 528

Liang, E.-W., Zhang, B.-B., \& Zhang, B. 2007, ApJ, 670, 565

Lindner, C. C., Milosavljevic, M., Couch, S. M., \& Kumar, P. 2010, ApJ, 713, 800

Lyons, N., et al. 2009, MNRAS, 402, 705

Lyutikov, M. 2009, Proc. Shocking Universe Meeting, Venice (arXiv:0911.0349)

Metzger, B. D. 2010, in ASP Conf. Proc. Frank N. Bash Symp.: New Horizons in Astronomy, ed. L. Stanford et al. (San Francisco, CA: ASP) (arXiv:1001.5046)

Metzger, B. D., Thompson, T. A., \& Quataert, E. 2007, ApJ, 659, 561

Nousek, J. A., et al. 2006, ApJ, 642, 389

Owen, B. J., et al. 1998, Phys. Rev. D, 58, 084020

Paczynski, R. 1992, Acta Astron., 42, 145

Rees, M. J., \& Mészáros, P. 1998, ApJ, 496, L1

Rhoads, J. E. 1997, ApJ, 487, L1

Sá, P. M. 2004, Phys. Rev. D, 69, 084001

Sá, P. M., \& Tomé, B. 2005, Phys. Rev. D, 71, 044007

Sá, P. M., \& Tomé, B. 2006, Phys. Rev. D, 74, 044011

Sari, R., Piran, T., \& Narayan, R. 1998, ApJ, 497, L17

Thompson, C. 1994, MNRAS, 270, 480

Troja, E., et al. 2007, ApJ, 665, 599

Usov, V. V. 1992, Nature, 357, 472

Usov, V. V. 1994, MNRAS, 267, 1035

Willingale, R., et al. 2007, ApJ, 662, 1093

Xu, R. X., Wang, H. G., \& Qiao, G. J. 2002, Chin. J. Astron. Astrophys., 2, 533

Yu, Y. W., Cao, X. F., \& Zheng, X. P. 2009a, Res. Astron. Astrophys., 9, 1024

Yu, Y. W., Cao, X. F., \& Zheng, X. P. 2009b, ApJ, 706, L221

Yu, Y. W., \& Dai, Z. G. 2007, A\&A, 470, 119

Zhang, B., \& Mészáros, P. 2001, ApJ, 552, L35

Zhang, B., et al. 2006, ApJ, 642, 354

Zhang, D., \& Dai, Z. G. 2008, ApJ, 683, 329

Zhang, D., \& Dai, Z. G. 2009, ApJ, 703, 461 